In Cres. Vol. $3 N^{\circ} 1: p p .71-85,2012$

\title{
GESTIÓN DE LA INTELIGENCIA EMOCIONAL EN EL NIVEL DIRECTIVO DE LA UNIVERSIDAD*
}

\author{
MANAGEMENT OF EMOTIONAL INTELLIGENCE IN \\ THE MANAGEMENT LEVEL OF THE UNIVERSITY
}

Tatiana Isabel Alvarez Becerra ${ }^{l}$

\section{RESUMEN}

La presente investigación se efectuó en el año 2011, en la Universidad Nacional de Trujillo y la Universidad Señor de Sipán de Chiclayo, Perú, con la finalidad de determinar ¿en qué medida el personal directivo de la Universidad Nacional de Trujillo y de la Universidad Particular Señor, de Sipán, Chiclayo, gestiona su inteligencia emocional en el trabajo? y ¿en qué medida influye esta gestión en la satisfacción laboral de sus colaboradores? La investigación es de tipo descriptivo y la muestra con la que se trabajó en ambas universidades fue de 448 personas de las cuales, 224 eran jefes y 224 , colaboradores directos de los mismos. La investigación determinó que la gestión de la inteligencia emocional en los directivos influye directamente en la satisfacción laboral de sus colaboradores en ambas universidades. Así mismo, se encontró que los directivos de la UNT presentan un nivel mayor de autoestima, locus de control interno, asertividad y satisfacción laboral, en comparación con los directivos de la USS.

PALABRAS ClAVE: Inteligencia emocional, Autoestima, Locus de control, Asertividad, Manejo de conflictos y satisfacción laboral.

* Recibido: 24 de enero del 2012; aceptado: 14 de junio del 2012.

1 Doctorado en Planificación y Gestión. Maestría en Gerencia de Servicios de Salud. Magíster en Ciencias de la Educación Superior con mención en Docencia Universitaria e Investigación. Consultora y Capacitadora a Núcleos Ejecutores por el Programa de Apoyo Social PAS, en Gobierno Regional La Libertad - Trujillo - Perú. 


\begin{abstract}
This descriptive research was conducted in the Universidad Nacional de Trujillo (UNT) and the Universidad Señor de Sipan (USS) in Chiclayo - Peru in the year 2011, in order to determine in what extent the managers of these two universities manage their emotional intelligence at work and how this influences their management on job satisfaction of their partners. The sample taken from both universities consisted of 448 people; 224 were heads of departments and 224 collaborators. The study found that emotional intelligence in leadership management, influences directly the collaborators's job satisfaction in both universities. Also, the study found that managers of the UNT present higher level of selfesteem, locus of internal control, assertiveness and job satisfaction, compared with managers of the USS.
\end{abstract}

KEY WORDS: Emotional intelligence, Self-esteem, Locus of control, Assertiveness, Conflict management and job satisfaction.

\title{
INTRODUCCIÓN
}

El término Inteligencia Emocional se refiere a la capacidad humana de sentir, entender, controlar y modificar estados emocionales en uno mismo y en los demás. Inteligencia emocional no es ahogar las emociones, sino dirigirlas y equilibrarlas. Disponemos de opciones para manejar las emociones. Ellas no son algo que tengamos dentro o fuera, sino que deben verse en términos de relaciones y esas relaciones se pueden manejar. Las emociones van a aparecer cuando haya una acción parcial o totalmente bloqueada; cuando no tengamos el control parcial o total de la situación; o cuando no podamos involucrarnos en la acción.

Para manejar las emociones debemos, en primer lugar, tener plena conciencia de lo que se está sintiendo, en qué circunstancias y a qué está asociado. De la misma manera, podemos reconocer la emoción, definir la situación en que sentimos incomodidad, aceptarla como válida, tanto la emoción como la situación. En segundo lugar, aceptar la emoción y darle la bienvenida a ese "monstruo". Mientras más se hable sobre algo, menos importante se vuelve. Entender por qué la emoción está ahí, que la acción ha sido bloqueada y, al mismo tiempo, saber en qué acción involucrarse, decirnos a nosotros mismos: "ven, ansiedad, acompáñame para seguir haciendo lo que tengo que hacer, yo sabía que ibas a aparecer". Así podremos tener la emoción a nuestro servicio y alcanzar nuestros objetivos. Si repetimos este mensaje, aun cuando la emoción siga ahí, resultará menos agobiante. 


\section{LA INTELIGENCIA EMOCIONAL EN EL TRABAJO}

El autor de la inteligencia emocional, Daniel Goleman, ${ }^{1}$ determina que las condiciones intelectuales no son la única garantía de éxito en el ámbito profesional del trabajo, sino tan sólo un factor que, unido a las necesidades emocionales cubiertas del personal como equipo, desarrollará el desempeño y los resultados de todo líder y trabajador, motivándolo emocionalmente a ser productivo. Una vez que una persona entra en una organización para que pueda dar lo mejor de sí, hacer bien su trabajo, que no sólo dé su talento, sino que además, lo haga con entusiasmo y compromiso, se necesitan:

Primero, que tenga sus útiles de trabajo y que sepa qué es lo que tiene que hacer;

Segundo, que sepa cómo hacerlo;

Tercero, que sienta que lo que está haciendo tiene un valor significativo; que él está contribuyendo y que se le reconozca por ello afectivamente.

El gerente puede ser muy competente, pero si no le da importancia a la gente que trabaja a su alrededor; si no reconoce sus esfuerzos; si ni siquiera le comunica que espera que hagan un buen trabajo, y que los va a ayudar en ese trabajo, lo que está generando en los demás son expectativas negativas y éstos se van a sentir muy incómodos, sentirán que no podrán hacerlo y cada vez que interactúen con el gerente como supervisor se van a sentir muy mal. Las competencias emocionales más decisivas en el éxito de los líderes y sus empresas, se clasifican en cuatro categorías, contando con varias competencias cada una para presentar así, las 20 Competencias Emocionales Claves, las cuales mencionaremos y comentaremos a continuación:

Autoconciencia: La habilidad para reconocer y comprender los propios estados emocionales, sentimientos, rasgos, así como su efecto en las demás personas. Las competencias que se miden y desarrollan en esta categoría son: la autoconfianza (autoestima), la capacidad para despertar estados emocionales alegres y llenos de buen humor.

Autorregulación: La habilidad para controlar y redireccionar impulsos y estados emocionales negativos, unido a la capacidad para suspender juicios y pensar antes de actuar. Las competencias que se miden y desarrollan en esta categoría son: autocontrol, confiabilidad (optimismo), conciencia, adaptabilidad, orientación a resultados e iniciativa.

Empatía: Las habilidades para sentir y palpar las necesidades de otros y 
de la propia organización, unida a la apertura para servir y cubrir las inquietudes de quienes le rodean. En esta categoría se miden y desarrollan: la empatía, la conciencia organizacional y la orientación al servicio (asertividad).

Socialización: Engloba el dominio de estrategias y formas de relacionarse afectiva y efectivamente con las demás personas, creando redes de relaciones, construyendo climas agradables, abiertos y efectivos en sus conversaciones. Las competencias en esta categoría son: desarrollo de persona, liderazgo, influencia, comunicación, gerencia del cambio, manejo de conflictos, construcción de redes y la cooperación en equipo.

Para efectos de la presente investigación, tomaremos como referencia una competencia emocional de cada categoría:

\section{a. Autoconciencia: Autoestima}

Es la experiencia personal de sentirse apropiado para la vida y las exigencias y retos que ella presenta. Es la disposición positiva de vivir de acuerdo con las posibilidades propias de crecimiento y desarrollo definiendo y alcanzando los propósitos. Por lo tanto, autoestima es la evaluación que hago de mi persona; no es la evaluación de un éxito o un fracaso particular, ni tampoco la evaluación de alguna habilidad o conocimiento puntual. Si tu autoestima depende de quién te quiere, de quién te tiene miedo, del dinero, del celular, del carro, de la posición social, etc., estás enfatizando aspectos pasajeros externos e inconsistentes.

\section{b. Autorregulación: Optimismo}

Es el arte de vivir, el hombre es, al mismo tiempo, artista y objeto de su arte, es el escultor y el mármol. Los individuos optimistas, confrontados por situaciones adversas las perciben como un reto y, por lo tanto, movilizan mayores recursos. Un concepto complementario al de optimismo es el locus de control. El locus de control es la percepción que tiene el individuo de cuánto control personal cree tener con relación a los eventos que afectan su vida; en otras palabras, en la relación que existe entre el esfuerzo ejercido y el resultado de ese esfuerzo en alcanzar los objetivos deseados. La palabra locus significa en griego "énfasis". El locus de control se divide en locus de control externo y locus de control interno. Los individuos con locus de control externo creen que tienen poco o ningún control sobre los eventos que afectan su vida; por lo tanto, no perciben que existe una relación entre su esfuerzo y el resultado obtenido. Estos son individuos que hablan de la siguiente manera: "El profesor me reprobó", "tuve mala suerte en mi matrimonio", "la felicidad es cosa del destino". Los 
individuos con locus de control interno ven una relación directa entre su esfuerzo y el resultado. Pueden expresarse diciendo: "Yo puedo decidir la dirección de mi vida", "si no salí bien en la prueba es porque no me preparé de acuerdo con las necesidades del examen y la materia; la próxima vez tengo que hacer un esfuerzo diferente", "la felicidad en mi vida es una decisión personal y depende de mi esfuerzo".

\section{c. Empatía: Asertividad}

Es la habilidad de expresar nuestras emociones y pensamientos, facilitando actuar en pro de nuestros mejores intereses y derechos, sin infringir o negar los de los demás. Además, asertividad también es la posibilidad de expresar espontáneamente tus gustos personales e intereses, hablar sobre ti mismo sin cohibirte, aceptar cumplidos cómodamente, no estar de acuerdo con alguien abiertamente, pedir aclaratorias y decir No.

\section{d. Socialización: Manejo de conflictos}

Siempre que existen desacuerdos en una situación social sobre asuntos importantes o que los antagonismos crean fricciones entre los individuos o los grupos, ocurre un conflicto. Los gerentes y los líderes de equipo pueden invertir un tiempo considerable para enfrentar conflictos, incluyendo aquéllos en los cuales ellos participan directamente como uno de los principales actores. En otras situaciones, actúan como mediadores, o como un tercero cuyo trabajo consiste en resolver los conflictos que existen entre otras personas.

Goleman ${ }^{1}$ propone que no es posible desarrollar las competencias emocionales a través del adiestramiento con cursos cortos que afirman y garantizan la posibilidad de ser inteligente emocionalmente con sólo un taller de uno o dos días.

Él afirma que es posible lograrlo con la presencia de un "coach" especializado en el seno de la empresa u organización donde interactúan las personas que desean contar con las mencionadas competencias. Goleman sugiere y cree en los programas largos (mínimo de 6 meses), en los cuales los participantes se ven motivados y exigidos a autoobservarse en su interacción con otros, contemplan la presencia de un "coach" o facilitador que actúa como espejo del comportamiento y además, con sesiones de aprendizaje y práctica de las competencias en la vida real del participante. Todo esto basado en un detallado diagnóstico validado, que permita determinar el perfil deseado, el perfil actual y las acciones para reducir el "gap" entre estos dos perfiles. Una persona puede tener miles de actitudes, pero el comportamiento organizacional se centra en un número limi- 
tado de actitudes relacionadas con el trabajo. Éstas capturan las evaluaciones positivas o negativas que los empleados mantienen acerca de sus jefes. Estas actitudes se han centrado en 3 principales:

\section{a. Satisfacción en el trabajo}

Se refiere a la actitud general de un individuo hacia su empleo. Una persona con alto nivel de satisfacción mantiene actitudes positivas hacia el trabajo, mientras que una persona insatisfecha mantiene actitudes contrarias. Cuando la gente habla de las actitudes del empleado, a menudo a lo que se refiere es a la satisfacción en el trabajo. La satisfacción en el trabajo es un conjunto de sentimientos favorables o desfavorables con los que los empleados perciben su trabajo.

La satisfacción en el trabajo también es parte de la satisfacción en la vida. La naturaleza del medio fuera del trabajo influye en los sentimientos que se tienen en el empleo. En el mismo sentido, debido a que el trabajo es una fuente importante de la vida, la satisfacción en éste influye en la satisfacción general de la vida. ${ }^{3}$

\section{b. Compromiso con el trabajo}

Este término mide el grado en el cual una persona se identifica sociológicamente con su trabajo y considera que su nivel de desempeño percibido es importante para valorarse a sí mismo. ${ }^{4}$

Los empleados con un alto nivel de compromiso con el trabajo se identifican sobremanera con el trabajo y en realidad les importa la clase de trabajo que hacen. La participación en el empleo es el grado en que los trabajadores se involucran en sus puestos, invierten tiempo y energía en ellos y ven al trabajo como una parte central de sus vidas en general; pocas veces llegan tarde o faltan, están dispuestos a trabajar muchas horas y tratarán de alcanzar niveles altos de desempeño.

\section{c. Compromiso organizacional}

Se define como un estado en el cual un empleado se identifica con una organización en particular y con sus metas; el trabajo significa identificarse con el trabajo específico de uno, mientras que un alto compromiso organizacional significa identificarse con el servicio personal a la organización. El compromiso organizacional es el nivel en que un empleado se identifica con la organización y desea seguir participando activamente en ella. Como una gran fuerza magnética que atrae un objeto metálico hacia otro, es un indicador de la disposi- 
ción del empleado a quedarse con una organización en el futuro. Con frecuencia refleja la creencia que tiene el trabajador en la misión y metas de la compañía, la disposición para dedicar esfuerzo para lograrlas y las intenciones para seguir trabajando allí. Los trabajadores comprometidos con la organización generalmente tendrán un buen historial de asistencia, apego gustoso a las políticas de la compañía e índices más bajos de rotación del personal. ${ }^{3}$

\section{EL EFECTO DE LA SATISFACCIÓN EN EL TRABAJO SOBRE EL DESEMPEÑO DEL EMPLEADO}

El interés de los gerentes en la satisfacción y en el trabajo tiende a centrarse en sus efectos sobre el desempeño del empleado. Los investigadores han reconocido este interés, así que concentramos un gran número de estudios que se han designado para evaluar el impacto de la satisfacción en el trabajo sobre la productividad del empleado, el ausentismo y la rotación. Las primeras opiniones sobre la relación entre la satisfacción y el desempeño puede resumirse esencialmente en el enunciado: "un trabajador feliz es un trabajador productivo". Los estudios sobre el tema de la satisfacción-productividad indican que la conclusión más válida es que la productividad lleva a la satisfacción, y no al contrario. ${ }^{5}$

Por otro lado, es necesario precisar que la insatisfacción del empleado se expresa de diversas formas; por ejemplo, en lugar de que renuncien, los empleados pueden quejarse, ser insubordinados, robar propiedad de la organización o aminorar sus responsabilidades de trabajo. ${ }^{6}$ Según Farrel, ${ }^{7}$ existen cuatro respuestas de insatisfacción:

- Salida: insatisfacción dirigida hacia el abandono de la organización; incluye el buscar una nueva posición, así como también la renuncia.

- Expresión: tratar activa y constructivamente de mejorar las condiciones, incluyendo las sugerencias de mejoras, la discusión de los problemas con superiores y algunas formas de actividad sindical.

- Lealtad: esperar de manera pasiva, pero con optimismo, a que mejoren las condiciones. Incluye hablar a favor de la organización en respuesta de la crítica externa y confiar en que la organización y su administración "hacen lo correcto".

- Negligencia: esperar pasivamente que empeoren las condiciones, incluyendo el ausentismo crónico o la impuntualidad, el esfuerzo reducido y una tasa mayor de error. 
Es importante fomentar el desarrollo de las habilidades, capacidades y disposición de los colaboradores para ejecutar con gracia, amor, paciencia y tolerancia las tareas, funciones, toma de decisiones en el organismo, empresa o institución donde sirven, como entes receptores y trasmisores de valores humanos universales. Estos valores significan cambios cualitativos importantes dentro de cualquier sociedad, pueblo o nación, y hacer que la sociedad sea orientada hacia el cambio y la transformación social para obtener como producto final el pleno desarrollo de la personalidad y el logro de un hombre sano, culto, critico y apto para convivir y funcionar en una sociedad democrática, justa y libre basada en la familia como célula fundamental y en la valorización del trabajo, capaz de participar activa, consciente y solidariamente con los procesos de transformación social, consustanciados en los valores de la identidad nacional y con la comprensión, tolerancia, convivencia y actitudes que favorezcan el fortalecimiento de la paz entre las naciones y los vínculos de integración y solidaridad. ${ }^{8}$

\section{MATERIAL Y MÉTODOS}

1. MATerial

1.1. Población: la población estuvo constituida por 488 personas de ambas universidades, de las cuales 244 eran directivos y 244 , asistentes administrativos.

1.2. Muestra: la muestra total ascendió a 224 personas en ambas universidades, distribuida de la manera siguiente:

Universidad Nacional de Trujillo: 76 directivos y 76 colaboradores. Universidad Señor de Sipán: 36 directivos y 36 colaboradores.

2. MÉTODO

2.1. Tipo de estudio: descriptivo.

2.2. Diseño de investigación: el diseño de la investigación es no experimental transeccional (de corte transversal) descriptivo simple.

\begin{tabular}{|c|c|}
\hline G.I.E. & S.L. \\
\hline Autoestima & \\
Optimismo & Satisfacción Laboral \\
Asertividad & \\
Manejo de conflictos & \\
\hline
\end{tabular}

Esquema del Diseño Descriptivo Simple. 
2.3. Variables y operativización de variables

\begin{tabular}{|c|c|c|c|}
\hline Variables & Nombre & $\begin{array}{l}\text { Definición } \\
\text { operacional }\end{array}$ & Indicadores \\
\hline \multirow{4}{*}{$\begin{array}{l}\text { VARIABLE } \\
\text { INDEPENDIENTE }\end{array}$} & \multirow{4}{*}{$\begin{array}{l}\text { GESTIÓN DE LA } \\
\text { INTELIGENCIA } \\
\text { EMOCIONAL }\end{array}$} & AUTOESTIMA & $\begin{array}{l}0-10 \text { puntos: } \quad \text { Muy baja autoestima. } \\
11-15 \text { puntos: } \quad \text { Baja autoestima. } \\
16 \text { puntos: } \quad \text { Autoestima promedio } \\
17-22 \text { puntos: } \quad \text { Alta autoestima. } \\
23-25 \text { puntos: } \\
\text { Muy alta autoestima. }\end{array}$ \\
\hline & & OPTIMISMO & $\begin{array}{l}\text { Locus de control interno. } \\
\text { Locus de control externo. }\end{array}$ \\
\hline & & ASERTIVIDAD & $\begin{array}{l}\text { Asertivo. } \\
\text { No asertivo. }\end{array}$ \\
\hline & & $\begin{array}{l}\text { MANEJO DE } \\
\text { CONFLICTOS }\end{array}$ & $\begin{array}{l}\text { Forzar. } \\
\text { Evitar. } \\
\text { Transigir. } \\
\text { Complacer. }\end{array}$ \\
\hline \multirow[b]{2}{*}{$\begin{array}{l}\text { VARIABLE } \\
\text { DEPENDIENTE }\end{array}$} & \multirow[b]{2}{*}{$\begin{array}{l}\text { SATISFACCIÓN } \\
\text { LABORAL }\end{array}$} & $\begin{array}{l}\text { TIPO DE } \\
\text { SATISFACCIÓN }\end{array}$ & $\begin{array}{l}\text { Satisfecho. } \\
\text { No satisfecho }\end{array}$ \\
\hline & & $\begin{array}{l}\text { NIVEL DE } \\
\text { SATISFACCIÓN }\end{array}$ & $\begin{array}{l}\text { Alta. } \\
\text { Conveniente. } \\
\text { Problemas. } \\
\text { Reencaminar. }\end{array}$ \\
\hline
\end{tabular}

\subsection{Instrumentos de recolección de datos}

Cuestionario: Cuestionario asertivo de Lazarus (empatía), Cuestionario de Locus de Control de Seeman y Evan (optimismo).

Encuesta: Encuesta de satisfacción laboral.

Inventario: Inventario de autoestima, de Cooper.

Test: Manejo de conflictos.

Se administraron cinco instrumentos a los colaboradores: uno para medir su satisfacción laboral; y cuatro instrumentos, para medir la empatía, el optimismo, la autoestima y el estilo para resolver conflictos de sus jefes.

\subsection{Análisis estadístico de datos}

La información obtenida fue analizada, interpretada y clasificada en cuadros estadísticos. Para determinar la dependencia de las variables, se empleó la prueba del Ji cuadrado. 


\section{RESULTADOS}

Luego de la realización de la investigación y de haber administrado y tabulado los diversos instrumentos, se encontraron los siguientes resultados:

El 98\% de los directivos de la USS están en un nivel muy alto y alto de autoestima, mientras que en la UNT ambos niveles representan el $48 \%$ en los directivos. Por otro lado, en la UNT el $17 \%$ de los colaboradores presenta un nivel bajo y muy bajo de autoestima, en la USS los colaboradores no presentan porcentajes en este indicador (Tabla 01).

Tabla 01

DISTRIBUCIÓN PORCENTUAL DE LOS NIVELES DE AUTOESTIMA

EN EL PERSONAL DIRECTIVO DE LA UNT Y LA USS

\begin{tabular}{|c|c|c|c|c|c|c|c|c|c|c|c|c|}
\hline \multirow{3}{*}{ Universidad } & \multicolumn{10}{|c|}{ Autoestima } & \multirow{2}{*}{\multicolumn{2}{|c|}{ Total }} \\
\hline & \multicolumn{2}{|c|}{ Muy Alta } & \multicolumn{2}{|c|}{ Alta } & \multicolumn{2}{|c|}{ Promedio } & \multicolumn{2}{|c|}{ Baja } & \multicolumn{2}{|c|}{ Muy Baja } & & \\
\hline & $\mathbf{N}^{0}$ & $\%$ & $\mathbf{N}^{\mathbf{0}}$ & $\%$ & $\mathbf{N}^{0}$ & $\%$ & $\mathbf{N}^{0}$ & $\%$ & $\mathrm{~N}^{\mathbf{0}}$ & $\%$ & $\mathrm{~N}^{\mathrm{o}}$ & $\%$ \\
\hline UNT & 39 & 30 & 23 & 18 & 15 & 11 & 8 & 6 & 15 & 11 & 76 & 100 \\
\hline USS & 15 & 42 & 20 & 56 & 1 & 2 & 0 & 0 & 0 & 0 & 36 & 100 \\
\hline
\end{tabular}

Fuente: Encuesta.

Elaborado por: La autora.

El 93,4\% del personal directivo de la UNT presenta un locus de control interno, mientras que en la USS este indicador, representa el $75 \%$ de la población (Tabla 02).

Tabla 02

DISTRIBUCIÓN PORCENTUAL DEL TIPO DE LOCUS DE CONTROL EN EL PERSONAL DIRECTIVO DE LA UNT Y LA USS

\begin{tabular}{|c|c|c|c|c|c|c|}
\hline \multirow{3}{*}{ Universidad } & \multicolumn{4}{|c|}{ Locus de control } & \multirow{2}{*}{\multicolumn{2}{|c|}{ Total }} \\
\hline & \multicolumn{2}{|c|}{ Externo } & \multicolumn{2}{|c|}{ Interno } & & \\
\hline & $\mathbf{N}^{\mathbf{0}}$ & $\%$ & $\mathbf{N}^{0}$ & $\%$ & $\mathbf{N}^{\mathbf{o}}$ & $\%$ \\
\hline UNT & 5 & 6,6 & 71 & 93,4 & 76 & 100 \\
\hline USS & 9 & 25,0 & 27 & 75,0 & 36 & 100 \\
\hline
\end{tabular}

Fuente: Encuesta.

Elaborado por: La autora.

Es notorio resaltar que el $73,7 \%$ de los directivos de la UNT son asertivos en sus relaciones interpersonales; mientras que en la USS este mismo indicador representa el 52,8\% (Tabla 03). 
Tabla 03

DISTRIBUCIÓN PORCENTUAL DEL TIPO DE ASERTIVIDAD

EN EL PERSONAL DIRECTIVO DE LA UNT Y LA USS

\begin{tabular}{|c|c|c|c|c|c|c|}
\hline \multirow{3}{*}{ Universidad } & \multicolumn{4}{|c|}{ Asertividad } & & \\
\hline & \multicolumn{2}{|c|}{ Asertivo } & \multicolumn{2}{|c|}{ No asertivo } & \multicolumn{2}{|c|}{ Total } \\
\hline & $\mathrm{N}^{\mathbf{0}}$ & $\%$ & $\mathbf{N}^{\mathbf{0}}$ & $\%$ & $\mathbf{N}^{0}$ & $\%$ \\
\hline UNT & 56 & 73,7 & 20 & 26,3 & 76 & 100 \\
\hline USS & 19 & 52,8 & 17 & 47,2 & 36 & 100 \\
\hline
\end{tabular}

Fuente: Encuesta.

Elaborado por: La autora.

En los directivos de la UNT el estilo para manejar conflictos es "Forzar" con el 39,5\%; mientras que en la USS es el de "Complacer", representando el $25 \%$ del total (Tabla 04).

Tabla 04

DISTRIBUCIÓN PORCENTUAL DEL ESTILO PARA MANEJAR CONFLICTOS EN EL PERSONAL DIRECTIVO DE LA UNT Y LA USS

\begin{tabular}{|c|c|c|c|c|c|c|c|c|c|c|c|c|}
\hline \multirow{3}{*}{ Universidad } & \multicolumn{10}{|c|}{ Manejo de conflictos } & & \\
\hline & \multicolumn{2}{|c|}{ Forzar } & \multicolumn{2}{|c|}{ Evitar } & \multicolumn{2}{|c|}{ Transigir } & \multicolumn{2}{|c|}{ Complacer } & \multicolumn{2}{|c|}{ Resolver } & \multicolumn{2}{|c|}{ Total } \\
\hline & $\mathbf{N}^{0}$ & $\%$ & $\mathbf{N}^{0}$ & $\%$ & $\mathbf{N}^{0}$ & $\%$ & $\mathrm{~N}^{\mathbf{0}}$ & $\%$ & $\mathbf{N}^{\mathbf{0}}$ & $\%$ & $\mathbf{N}^{\circ}$ & $\%$ \\
\hline UNT & 30 & 39,5 & 17 & 22,4 & 13 & 17,1 & 4 & 5,3 & 12 & 15,8 & 76 & 100 \\
\hline USS & 6 & 16,7 & 7 & 19,4 & 7 & 19,4 & 9 & 25,0 & 7 & 19,4 & 36 & 100 \\
\hline
\end{tabular}

Fuente: Encuesta.

Elaborado por: La autora.

El 71,1\% de los colaboradores directos del personal directivo en la UNT se encuentra satisfecho en su trabajo, mientras que el mismo grupo en la USS, representa el 38,9\% (Tabla 05).

\section{Tabla 05}

DISTRIBUCIÓN PORCENTUAL DEL NIVEL DE SATISFACCIÓN LABORAL EN LOS COLABORADORES DIRECTOS DEL PERSONAL DIRECTIVO DE LA UNT Y LA USS

\begin{tabular}{|c|c|c|c|c|c|c|c|c|c|c|}
\hline \multirow{3}{*}{ Universidad } & \multicolumn{8}{|c|}{ Nivel de satisfacción laboral } & \multirow{2}{*}{\multicolumn{2}{|c|}{ Total }} \\
\hline & \multicolumn{2}{|c|}{ Alto } & \multicolumn{2}{|c|}{ Medio } & \multicolumn{2}{|c|}{ Bajo } & \multicolumn{2}{|c|}{ Muy bajo } & & \\
\hline & f & $\%$ & f & $\%$ & f & $\%$ & f & $\%$ & f & $\%$ \\
\hline UNT & 54 & 71,1 & 8 & 10,5 & 8 & 10,5 & 6 & 7,9 & 76 & 100 \\
\hline USS & 14 & 38,9 & 10 & 27,8 & 7 & 19,4 & 5 & 13,9 & 36 & 100 \\
\hline
\end{tabular}

Fuente: Encuesta.

Elaborado por: La autora. 


\section{DISCUSIÓN}

En cuanto a la variable autoestima y su relación con la satisfacción laboral, se corrobora con los manifestado por Abraham Maslow en su jerarquía de las necesidades humanas, donde describe que la autoestima es la necesidad de aprecio, la cual se divide en dos aspectos: el aprecio que se tiene uno mismo (amor propio, confianza, pericia, suficiencia, etc.), y el respeto y estimación que se recibe de otras personas (reconocimiento, aceptación, etc.). Al relacionar autoestima con satisfacción laboral, se puede apreciar un nivel significativo $(\mathrm{p}<0,01)$ entre estas variables, concluyendo que a mayor autoestima del directivo más satisfecho se encuentra el trabajador tanto a nivel organizacional como personal, lo cual se relaciona con lo estudiado por Loli \& Co. (1998), quienes realizaron una investigación relativa a la autoestima y los valores organizacionales en 105 gerentes de microempresas, encontrando correlación significativa entre autoestima y algunos valores organizacionales (lealtad, amor al trabajo, trabajo en equipo).

Por otro lado, el locus de control de los directivos influye en la satisfacción laboral de sus colaboradores; es decir, las creencias, ideas y expectativas que poseen los directivos de ambas universidades les permite evaluar o analizar las diferentes situaciones del campo de trabajo, facilitando al agrado o desagrado de las mismas según el valor subjetivo que el trabajador da a las consecuencias de su comportamiento, lo cual es reafirmado por Jhonson \& Luthan (1984), puesto que ellos afirman que el locus de control es aplicable a todas las áreas de la vida, sus implicaciones en el comportamiento, tanto para el trabajo como en la vida personal, puesto que las personas con un locus de control interno van a controlar su propio ambiente comportándose de determinada manera para obtener el control de las situaciones. Los directivos de ambas universidades presentaron un locus de control interno: UNT 93,4\% y USS $75 \%$ (ver tabla 02). Esto significa que éstos creen en sus propios logros, habilidades y capacidades, mostrándose capaces de desenvolverse en cualquier ámbito laboral, lo cual es reafirmado por Rotter, quien refiere que las personas con tendencia de autocontrol interno presentan capacidad de discernimiento o autovaloración ante las situaciones cotidianas; creen en su propia capacidad para lograr lo que se proponen mediante su esfuerzo y habilidades mostrándose capaces de desenvolverse en cualquier ámbito laboral; por ende, se creen responsables de sus propios logros o fracasos. Cuando relacionamos la variable locus de control con satisfacción laboral, se puede apreciar un nivel significativo $(\mathrm{p}<0,01)$ entre estas variables debido que los tra- 
bajadores creen que sus propias capacidades y habilidades consolidarán un desempeño exitoso. Esto es reafirmado por Whittaker\& Chang (1999), quienes al someter a investigación una muestra de 50 trabajadores observaron que, en la medida en que el trabajador se da cuenta que son sus propios resultados lo que incrementa su productividad en sus actividades laborales, se logra por medio de esfuerzo constante y no la suerte o preferencias, podrán adoptar con mayor facilidad comportamientos y actitudes optimas que les permitirá cumplir mejor con su responsabilidades.

Asimismo, la variable asertividad también influye en la satisfacción de los colaboradores, dado que es un concepto aportado por la psicología moderna a la comprensión y mejora de nuestras relaciones sociales. Los directivos del estudio, presentaron niveles de $73,7 \%$ en la UNT y $52,8 \%$ en la USS. Según lo manifestado por Pérez (psicólogo de Asimet), la asertividad es significativa, relevante en el mundo del trabajo, entre otras habilidades importantes dignas de cuidar y desarrollar. En la relación asertividadsatisfacción laboral se puede apreciar un nivel significativo $(\mathrm{p}<0,01)$ entre estas variables debido que los trabajadores consideran que los directivos deben hacer valer sus derechos, pero sin atropellar los ajenos, lo que conlleva a buen clima organizacional respetando espacios e ideas, generando por ende, satisfacción personal y organizacional.

En cuanto a la variable de manejo de conflictos, el estudio arrojó que en la UNT el principal estilo es el de forzar $(39,5 \%)$, mientras que en la USS es transigir y resolver $(38,8 \%)$. El estilo de resolver conflictos juega un papel preponderante en la satisfacción laboral de los colaboradores, pues según lo manifestado por Robbins (1999), el conflicto puede ser un problema serio en cualquier organización y puede dañar el desempeño de la organización y llevar a la pérdida de muchos buenos empleados. En la relación de ambas variables, manejo de conflictos-satisfacción laboral, se puede apreciar un nivel significativo $(\mathrm{p}<0,01)$, lo que quiere decir que a mejor manejo de conflictos mayor satisfacción laboral y viceversa.

\section{CONCLUSIONES}

1. Se corrobora la hipótesis planteada, ya que se demostró que la gestión de la inteligencia emocional, representada en los factores de autoestima, locus de control, asertividad y manejo de conflictos de los directivos de la UNT y la USS, repercute directamente en la satisfacción laboral de sus colaboradores 
directos, tal como lo arroja la prueba del Ji cuadrado, que denota claramente la relación entre una variable y otra.

2. En la Universidad Nacional de Trujillo, los directivos presentaron un nivel muy alto de autoestima, un locus de control interno, asertividad y empleo de un estilo de forzar para solucionar sus conflictos.

3. En la Universidad Señor de Sipán de Chiclayo, los directivos presentaron un nivel alto de autoestima, un locus de control interno, asertividad y empleo de estilos de transigir y resolver para solucionar sus conflictos.

4. Tanto en la Universidad Nacional de Trujillo como en la Universidad Señor de Sipán, de Chiclayo, los colabores directos de los directivos presentaron un alto nivel de satisfacción laboral.

5. Comparando entre los indicadores estudiados en ambas universidades, encontramos que los directivos de la UNT presentan un nivel mayor de autoestima, locus de control interno, asertividad y satisfacción laboral, en comparación con los directivos de la USS.

\section{REFERENCIAS BIBLIOGRÁFICAS}

1 Goleman, DANIEL. La Inteligencia Emocional. Madrid, Javier Vergara-Grupo Z., 1996.

2 GIL'ADÍ, DANIEL. Inteligencia Emocional en Práctica, (1 a Ed.), Colombia, McGraw Hill, 2000.

3 Davit, Keith and Newstrom, Jhon. Comportamiento Humano en el Trabajo. 3ra. Ed., México, McGraw Hill, 1997.

4 BlaU, G.J. y BOAL, K.L. Conceptualizing How Job Involvement and Organizational Commitment Affect Turnover and Absenteeism. Academy of Management Review, 1987.

5 GreENE, C.N. The Satisfaction Performance Controversy. Business Horizons, febrero, 1972.

6 Fisher, C.D. y LOCKE, E.A. The New Look in Job Satisfaction Research and Theory, 1994.

7 FARrel, D. Impact of Job Satisfaction, Investment Size, and Quality of Alternatives on Exit, Voice, Loyalty, and Neglect Responses to Job Dissatisfaction, A Cross-Legged Panel Study. San Francisco, 1990.

8 RobBins, Stephen P. Administración. México, Pearson, 1998.

9 ARveY, R.D. Genetics, Twins, and Organizational Behavior. Greenwich, 1994.

10 BAssett, G. The Case Against Job Satisfaction. Business Horizons, 1994.

11 Bilancio, Guillermo. Antióxido. 1ra. Ed., Brazil, Prentice, 2001.

12 Branden, Nathaniel. Cómo Mejorar su Autoestima. Buenos Aires, Paidós, 1997.

13 BRECLER, S.J. Empirical Validation of Affect, Behavior, and Cognition as District Components of Attitude. Journal of Personality and Social Psychology, 1984.

14 Cano, Bethel. La Ética, Arte de Vivir. Colombia, Paulinas, 2000. 
17 De Bono Edward. El Pensamiento Lateral, Manual de Creatividad. Argentina, Paidos Plural, 2000.

18 Dell, Twyla. La Motivación en el Trabajo. México, Trillas, 1991.

19 Feldman, D.C. y ARnOld, H.J. Personality Tipes y Career Patterns", Some Empirical Evidence on Holland's Model. Canadian Journal of Administrative Science, 1985.

20 Fournies, Ferdinand. Técnicas de Dirección de Personal. México, McGraw Hill, 1994.

21 GalluP, G. Employee Research, From Nice to Know to Need to Know. Personnel Journal, 1988.

22 García, M y Magaz, A. Aprendiendo a Comunicarse con Eficacia. España, CEPF, 1995.

23 Gardner, Howard. Estructura de la Mente. La Teoría de las Múltiples Inteligencias. México, FCE, 1987.

24 Gerhart, B. How Important are Dispositional Factors as Determinants of Job Satisfaction? Implications for Job Desing and Other Personnel Programs. Journal of Applied Psychology, agosto, 1987.

25 Hodson, R. Workplace Behaviors. Work and Ocupations, agosto, 1991.

26 KATZELL, R.A. How Job Satisfaction and Job Performance Are and Are nor Linked. Nueva York, 1992.

27 Loche, E.A. The Nature and Causes of Job Satisfaction. Chicago, Handbook of Industrial and Organizational Psycology, 1976.

28 LOLI P., A. LÓPEZ V., Ernestina. «Autoestima y valores en la calidad y la excelencia». Lima, Instituto de Investigaciones Psicológicas, UNMSM, 1998.

29 LYNE, G.E. How to Measure Employee Attitudes. Training and Development Journal, diciembre, 1989.

30 Mackay, M, Fanning, P. Técnicas de Autocontrol de la Conducta Emocional. Barcelona, Martínez Roca, 1987.

31 Powell, Jhon. Autoestima. México, Diana, 1997.

32 PRICE, J.L. Handbook of Organizational Measurement., Marshfield, 1986.

33 RobBins, Stephen. Comportamiento Organizacional 8va. Ed., México, Pearson, 1999.

34 Robbins, Stephen P. La Administración en el Mundo de Hoy. México, Pearson, 1998.

35 RocKeach, M. The Nature of Human Values. Nueva York, Free Press, 1973.

36 Segal, Jeanne. Su Inteligencia Emocional, Aprenda a incrementarla y a usarla. Barcelona, Grijalbo, 1999.

37 Senge, Peter. La Quinta Disciplina. México, Gránica, 1990.

38 Soto, EduARdo. Comportamiento Organizacional. $1^{\text {a }}$ Ed., México, Thompson, 2001.

39 Vargas de GonzÁlez, Gaby. La Imagen del Éxito. $1^{\text {a }}$ Ed., México, Mc Graw Hill, 1998.

Tatiana Isabel Alvarez Becerra

tab@upnorte.edu.pe 
